\title{
Numerical and Experimental Analysis of Circular-End Wire Bow-Tie Antennas Over a Lossy Ground
}

\author{
Andrian Andaya Lestari, Alexander G. Yarovoy, Member, IEEE, and Leo P. Ligthart, Fellow, IEEE
}

\begin{abstract}
This paper presents a numerical and experimental analysis of a wire bow-tie antenna situated horizontally near a lossy ground. The antenna is basically an array of identical wire dipoles having a common feed point and equal angular separation between two neighboring wires. This antenna is particularly suitable for ultrawide-band applications as it possesses a wideband property and allows a simple realization of resistive loading. It also provides a possibility to easily vary the antenna flare angle for the purpose of antenna matching or to adjust its footprint. In this work a theoretical model of the antenna is developed and verified experimentally. The wires are modeled as a tape-like structure by triangular patches and the antenna is analyzed using a mixed-potential integral equation formulation. Moreover, time-domain solutions are computed using the Fourier transformation, and a time-window technique is employed to calculate the antenna characteristic impedance. Using the model a comprehensive analysis of the antenna performance is carried out.
\end{abstract}

Index Terms-Bow-tie antennas, ground penetrating radar, mixed-potential integral equation, wire antennas.

\section{INTRODUCTION}

$\mathbf{S}$ OLID bow-tie antennas situated in free space as well as above the ground have been thoroughly investigated by many researchers [1]-[6]. Bow-tie antennas, which basically are the limiting case of biconical antennas [2], are attractive mainly due to their simplicity and wideband property which can be considerably improved by resistive loading [3]. Because of this, resistively loaded bow-tie antennas are widely employed in ultrawide-band applications such as impulse ground penetrating radar (GPR). It is our intention to focus on this particular application in this paper.

The most commonly used bow-tie antennas are the ones with straight ends, forming a triangular shape of the bow-tie arms [1], [4]-[6]. However, for transient applications of bow-tie antennas, bow ties with circular ends [3] demonstrate a better performance as reflections from the ends occur at the same instant. In contrast to straight-end bow-tie antennas, the ones with circular ends form fan-like structures. Especially for large flare angles, this leads to a pronounced difference in their transient behavior when their side-edge length is comparable to the spatial

Manuscript received March 15, 2002; December 14, 2002. This work was supported by the Dutch Technology Foundation (STW) under the projects "Improved Ground Penetrating Radar Technology" (1999-2000) and "Advanced Re-Locatable Multisensor System for Buried Landmine Detection" (2001-2002).

The authors are with the International Research Centre for Telecommunications-transmission and Radar (IRCTR), Delft University of Technology, Delft 2628 CD, The Netherlands.

Digital Object Identifier 10.1109/TAP.2003.820959 length of the pulse. In such a situation, straight-end bow-tie antennas suffer more from early arrival of end reflections, since the straight ends cause widening of the reflected pulses. This in turn might cause overlapping of the main pulse and end reflections. Contrary to this, circular-end bow-tie antennas do not suffer from widening of the reflected pulses, even for very large flare angles, and thus overlapping of the pulses can be more easily prevented. This aspect is particularly important to this work since some of the computations are carried out as a function of flare angle. It is for this reason that here we concentrate only on circular-end bow-tie antennas and any bow-tie term found in the rest of the paper refers to this type of bow tie.

In this work, solid bow-tie antennas are approximated using straight wires arranged in a unique configuration, in which the resulting structure is basically an array of several identical wire dipoles having a common feed point and equal angular separation between two neighboring wires, as shown in Fig. 1. This structure has a principal difference in shape and configuration with respect to the triangular wire bow-tie structure discussed by Smith, et al. in [7]. Furthermore, the authors have observed that a similar wire structure is employed by commercial GPR systems.

The analyzed wire bow-tie structure has several advantages in comparison with solid ones. A wire structure makes it easier to effectively implement a resistive loading for proper transmission of ultrawide-band pulses. Moreover, with a slight modification such a structure allows one to vary the bow-tie flare angle. It has been shown that the size and shape of the subsurface footprint of a GPR bow-tie antenna can be varied by changing the flare angle [8]. Due to this capability one can reduce ground clutter when the flare angle is adjusted so that the size and shape of the illuminated area in the ground is comparable to those of the targets. As a result, a significant improvement of imaging could be achieved.

The code employed in this work has been developed to analyze various types of GPR antennas, which are not limited to the antennas discussed in this paper. It makes use of the integral equation method, particularly the mixed-potential integral equation (MPIE) formulation $\mathrm{C}$ for problems in layered media by Michalski and Zheng [9], which is numerically solved by the method of moments (MoM) using Rao-Wilton-Glisson (RWG) basis functions [10]. By means of the equivalent radius formula [11], the antenna wires are modeled as tape-like structures using triangular patches. Furthermore, time-domain solutions are obtained by the Fourier transformation, and a time-window technique is applied to compute the characteristic impedance of the antenna by removing the pulses associated with internal reflections in the antenna. As a large number 


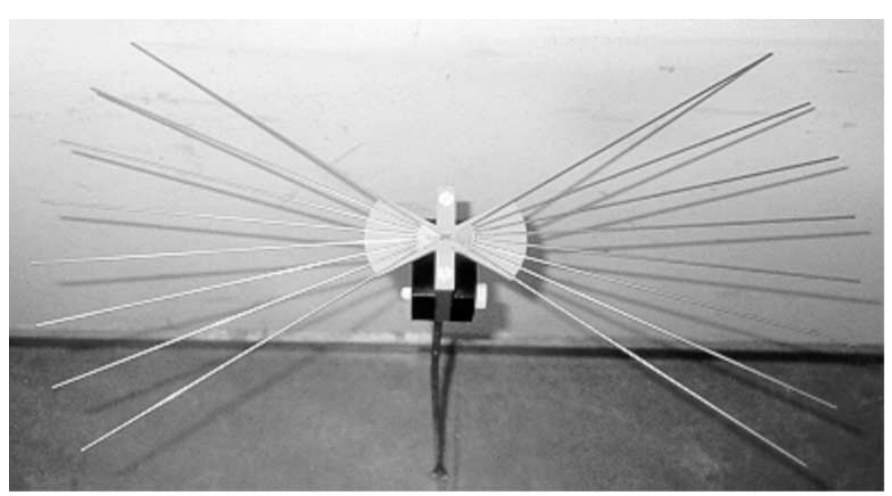

(a)

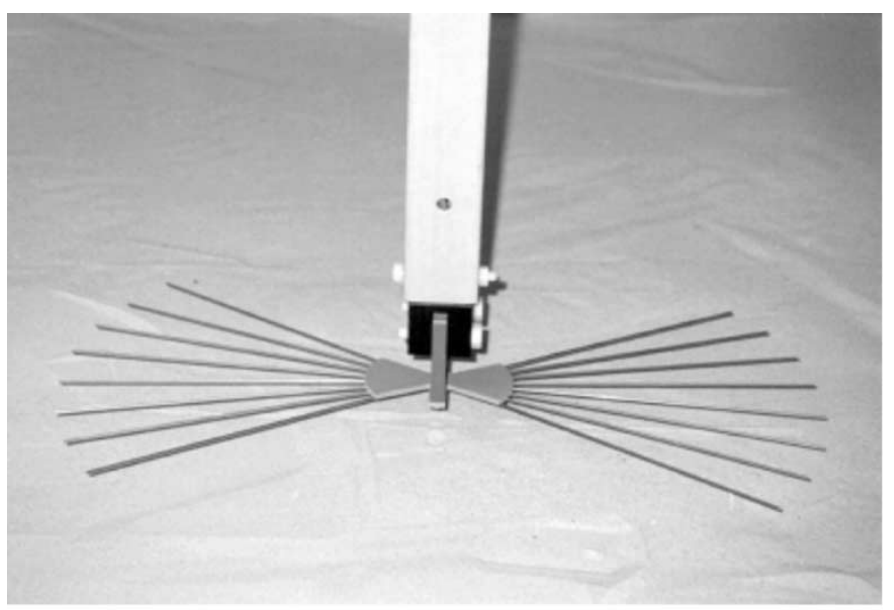

(b)

Fig. 1. (a) Experimental eight-element wire bow-tie antenna (wire diameter $=2 \mathrm{~mm}$, length $=50 \mathrm{~cm}$ ) with $10^{\circ}$ separation between neighboring elements, forming a flare angle of $70^{\circ}$. (b) The antenna is situated horizontally $5 \mathrm{~mm}$ above dry sand.

of frequency-domain computations are required to obtain an adequate time-domain resolution, we implement an efficient algorithm to prevent excessive computation time. This includes exploitation of antenna symmetry, efficient computations of impedance matrix elements, and a special treatment of integration over singularity. Moreover, numerical integrations of the slowly converging Sommerfeld integrands are accelerated using an interpolation scheme.

\section{NUMERICAL MODEL}

\section{A. Mixed-Potential Integral Equation}

Assuming the positive time dependence $e^{j \omega}$ the employed MPIE formulation by Michalski and Zheng [9] for problems in a planar two-layer medium which is unbounded in the $x$ and $y$ direction in Cartesian coordinates, can be written as

$\hat{n}_{m} \times \sum_{k=1}^{2}\left[j \omega \mathbf{A}^{m k}(\mathbf{r})+\nabla \phi^{m k}(\mathbf{r})\right]=\hat{n}_{m} \times \mathbf{E}_{m}^{\mathrm{I}}(\mathbf{r})$,

$\mathbf{r}$ on $S_{m}, m=1,2$ where the source point is in layer $k$, the observation point is in layer $m, \hat{n}_{m}$ is a unit vector normal to the surface $S_{m}, \mathbf{E}^{1}$ is the incident field, and the potentials are given by

$$
\begin{aligned}
\mathbf{A}^{m k}(\mathbf{r}) & =\int_{S_{k}} \overline{\mathbf{K}}_{A}^{m k}\left(\mathbf{r} \mid \mathbf{r}^{\prime}\right) \cdot \mathbf{J}\left(\mathbf{r}^{\prime}\right) d S^{\prime} \\
\phi^{m k}(\mathbf{r}) & =\int_{S_{k}} K_{\phi}^{m k}\left(\mathbf{r} \mid \mathbf{r}^{\prime}\right) q\left(\mathbf{r}^{\prime}\right) d S^{\prime}
\end{aligned}
$$

where primes denote source quantities and $q$ is the surface charge density. The surface current density is related to $q$ by the continuity equation

$$
\nabla \cdot \mathbf{J}=-j \omega q .
$$

In (2), $\overline{\mathbf{K}}_{\mathbf{A}}^{m k}$ is the dyadic Green's function given by

$$
\begin{aligned}
\overline{\mathbf{K}}_{A}^{m k}\left(\mathbf{r} \mid \mathbf{r}^{\prime}\right)= & (\hat{\mathbf{x}} \hat{\mathbf{x}}+\hat{\mathbf{y}} \hat{\mathbf{y}}) K_{x x}^{m k}+\hat{\mathbf{x}} \hat{\mathbf{z}} K_{x z}^{m k} \\
& +\hat{\mathbf{y}} \hat{\mathbf{z}} K_{y z}^{m k}+\hat{\mathbf{z}} \hat{\mathbf{x}} K_{z x}^{m k}+\hat{\mathbf{z}} \hat{\mathbf{y}} K_{z y}^{m k}+\hat{\mathbf{z}} \hat{\mathbf{z}} K_{z z}^{m k} .
\end{aligned}
$$

The expressions for the scalar Green's function $K_{\phi}^{m k}$ in (3) and the components $K_{x x}^{m k}, K_{x z}^{m k}, K_{y z}^{m k}, K_{z x}^{m k}, K_{z y}^{m k}, K_{z z}^{m k}$ in (5) are tabulated in [9]. In the present work we only use $K_{x x}^{m k}$ since the antenna is planar and situated horizontally.

In the employed MoM scheme the current density $\mathbf{J}$ on the antenna surface $S$ is approximated in terms of RWG basis functions $\mathbf{f}_{n}$ as

$$
\mathbf{J}(\mathbf{r}) \approx \sum_{n=1}^{N} I_{n} \mathbf{f}_{n}(\mathbf{r}), \quad \mathbf{r} \text { on } S
$$

where $\mathbf{r}$ is the position vector from the origin, $N$ is the number of basis functions and $I_{n}$ 's are the unknowns to be solved representing the antenna currents. The basis functions $\mathbf{f}_{n}$ are defined in a pair of adjacent triangles $n$ as specified by Rao et al. in [10]. Substituting (6) in (1) and applying point matching in the MoM scheme, one obtains an $N$ by $N$ system of linear equations as

$$
\left[Z_{p n}\right]\left[I_{n}\right]=\left[V_{p}\right]
$$

where

$$
\begin{aligned}
Z_{p n} & =l_{p}\left[\frac{j \omega}{2}\left(A_{p n}^{-}+A_{p n}^{+}\right)-\frac{1}{j \omega}\left(\Phi_{p n}^{-}-\Phi_{p n}^{+}\right)\right] \\
V_{p} & =\frac{l_{p}}{2}\left[\rho_{p}^{c+} \cdot \mathbf{E}_{m}^{\mathrm{I}}\left(\mathbf{r}_{p}^{c+}\right)+\rho_{p}^{c-} \cdot \mathbf{E}_{m}^{\mathrm{I}}\left(\mathbf{r}_{p}^{c-}\right)\right]
\end{aligned}
$$

with the superscripts + and - used to identify each triangle in the pair of adjacent triangles $p$ in which RWG basis functions $\mathbf{f}_{p}$ are defined, $l_{p}$ is the length of the common edge of the pair of triangles $p, \rho_{p}^{c \pm}$ is the vector between the free vertex and the centroid of each triangle in the pair of triangles $p, \mathbf{r}_{p}^{c \pm}$ is the position vector from the origin to the centroid of each triangle in the pair of triangles $p$, and $p, n=1,2 \ldots, N$. In (8) the potentials are given by

$$
\begin{aligned}
& A_{p n}^{ \pm}=\int_{S_{k}} \rho_{p}^{c \pm} \cdot \overline{\mathbf{K}}_{A}^{m k}\left(\mathbf{r}_{p}^{c \pm} \mid \mathbf{r}^{\prime}\right) \cdot \mathbf{f}_{n}\left(\mathbf{r}^{\prime}\right) d S^{\prime} \\
& \Phi_{p n}^{ \pm}=\int_{S_{k}} K_{\phi}^{m k}\left(\mathbf{r}_{p}^{c \pm} \mid \mathbf{r}^{\prime}\right) \nabla_{S}^{\prime} \cdot \mathbf{f}_{n}\left(\mathbf{r}^{\prime}\right) d S^{\prime}
\end{aligned}
$$

A voltage source model similar to that in [12] is used for excitation, in which a thin slot is assumed to exist along the 
common edge of the pair of triangles $p$ that represent the antenna terminal. A voltage difference is then prescribed across the slot, which in turn generates an impressed electric field within the slot. Thus, assuming there are no other excitations, in place of (9) we have [12]

$$
V_{p}=l_{p} V_{p}^{\text {source }}
$$

where $V_{p}^{\text {source }}$ is the prescribed voltage across the slot.

\section{B. Efficient Numerical Implementation}

As a large number of frequency-domain computations are required to guarantee sufficient resolution in the time domain, in this work we develop an efficient algorithm which allows typical GPR antennas over a lossy ground to be evaluated within a reasonable time. The algorithm is based on four factors: 1) efficient calculations of impedance matrix elements; 2) exploitation of antenna symmetry; 3) a special treatment of integration over singularity; and 4) an interpolation method for Sommerfeld integrals.

1) Efficient Calculations of Impedance Matrix Elements: Using RWG basis functions, the impedance matrix $Z_{p n}$ can be obtained most easily by calculating the matrix elements directly for each pair of basis functions. However, such a brute-force approach is inefficient, because the same value of vector and scalar potentials could appear in 3 different pairs of basis functions. To perform a much more efficient computation we use a method similar to that in [10], in which we need to calculate the magnetic vector potential

$$
A_{i}^{t u}=\int_{T_{k}^{u}} \rho_{t}^{c} \cdot \overline{\mathbf{K}}_{A}^{m k}\left(\mathbf{r}_{t}^{c} \mid \mathbf{r}^{\prime}\right) \cdot \mathbf{f}_{i}\left(\mathbf{r}^{\prime}\right) d S^{\prime}
$$

and the electric scalar potential

$$
\Phi_{i}^{t u}=\int_{T_{k}^{u}} K_{\phi}^{m k}\left(\mathbf{r}_{t}^{c} \mid \mathbf{r}^{\prime}\right) \nabla_{S}^{\prime} \cdot \mathbf{f}_{i}\left(\mathbf{r}^{\prime}\right) d S^{\prime}
$$

associated with the $i^{\text {th }}$ basis function on patch $u$ observed at the centroid of patch $t$, where $i=1,2,3, p=q=1,2, \ldots N_{T}$, with $N_{T}$ the number of triangular patches, and the integrals in (13) and (14) are evaluated over the triangular patch $T^{u}$ of the source.

2) Exploitation of Antenna Symmetry: The symmetrical geometry of bow-tie antennas can be exploited to reduce computation time. As an example, for horizontal bow ties with main axis on the $x$ axis, exploiting the symmetry with respect to the $x=0$ plane results in the reduction of matrix-fill time by a factor of two. To this end, we need to evaluate (13) and (14) only for source points on one of the two arms. Thus, in this case we have $t=1,2, \ldots, N_{T} / 2$ and $u=1,2, \ldots, N_{T} / 2$. The other half of the integrals can be obtained directly from the computed first half, as follows

$$
\begin{aligned}
A_{x i}^{t u} & =-A_{x i}^{t+N_{T} / 2 u-N_{T} / 2} \\
A_{y i}^{t u} & =A_{y i}^{t+N_{T} / 2 u-N_{T} / 2} \\
\Phi_{i}^{t u} & =\Phi_{i}^{t+N_{T} / 2 u-N_{T} / 2}
\end{aligned}
$$

for $t=1,2, \ldots, N_{T} / 2$ and $u=N_{T} / 2+1, N_{T} / 2+2, \ldots, N_{T}$, and

$$
\begin{aligned}
A_{x i}^{t u} & =-A_{x i}^{t-N_{T} / 2 u-N_{T} / 2} \\
A_{y i}^{t u} & =A_{y i}^{t-N_{T} / 2 u-N_{T} / 2} \\
\Phi_{i}^{t u} & =\Phi_{i}^{t-N_{T} / 2 u-N_{T} / 2}
\end{aligned}
$$

for $t=N_{T} / 2+1, N_{T} / 2+2, \ldots, N_{T}$ and $u=N_{T} / 2+1$, $N_{T} / 2+2, \ldots, N_{T}$. With more effort in bookkeeping, further reduction of matrix-fill time can of course be obtained by taking the symmetry with respect to the $y=0$ plane into account.

3) Special Treatment of Integration Over Singularity: The evaluation of the self-terms in the impedance matrix $Z_{p n}$ in (7) is the most time consuming since it involves singular integrands. The kernel $\overline{\mathbf{K}}_{A}$ in (13) and $K_{\phi}$ in (14) have $1 /\left|\mathbf{r}-\mathbf{r}^{\prime}\right|$ singularities, which for a given accuracy require more subdivisions to be properly evaluated by a numerical quadrature. To improve computation efficiency, in this work we introduce a special treatment to accelerate the evaluation of such singular integrands, which ultimately reduces the overall computation time significantly.

The most-widely used numerical quadrature techniques for triangular domains include the so-called three-point (quadratic), four-point (qubic) and seven-point (quintic) quadrature rules, in which the integrand is evaluated at respectively 3,4 , and 7 discrete points within a triangular domain [13]. For a singular integrand, only the quadratic quadrature rule is applicable because it is the only one that does not have an evaluation point at the centroid. Unfortunately, as singular integrands involve a rapidly varying function, generally in this case the quadratic quadrature will not provide sufficient accuracy. The traditional approach to circumvent this problem is by recursively subdividing the whole domain into smaller triangles, until the desired accuracy is reached. However, this approach leads to excessive computation time since a large number of subdivisions are required due to the rapidly varying integrand near the singularity. Therefore, only a limited number of subdivisions can usually be used and it has been shown that this approach is adequate only for far-field analysis [14]. For near-field analysis, in which the self-term elements of the impedance matrix must be accurately evaluated, one may extract the singularity from the integrand and integrate the remainder numerically [15]. As an alternative, here we introduce a simple yet effective approach to efficiently evaluate integrands with $1 /\left|\mathbf{r}-\mathbf{r}^{\prime}\right|$ singularities using the quadratic quadrature rule, which to our knowledge has never been reported by other investigators. The proposed technique, which is fully numerical, is more straightforward and simpler than the ones in [15] and [16] since no manipulation of the integrands is involved.

By noting that the $1 /\left|\mathbf{r}-\mathbf{r}^{\prime}\right|$ singularities in (13) and (14) are rapidly varying only in the region near the centroid of the triangular patch, we divide the triangular domain into two main triangles, indicated by triangles ABC and DEF shown in Fig. 2. The region outside the inner triangle (DEF) is recursively subdivided only for a few times. Typically, two or three subdivisions would be sufficient to evaluate this part of the integral due to its more slowly varying integrand. The most important part of this approach is the evaluation of the inner triangle that bounds the singularity. By taking into account that the integrand varies more 


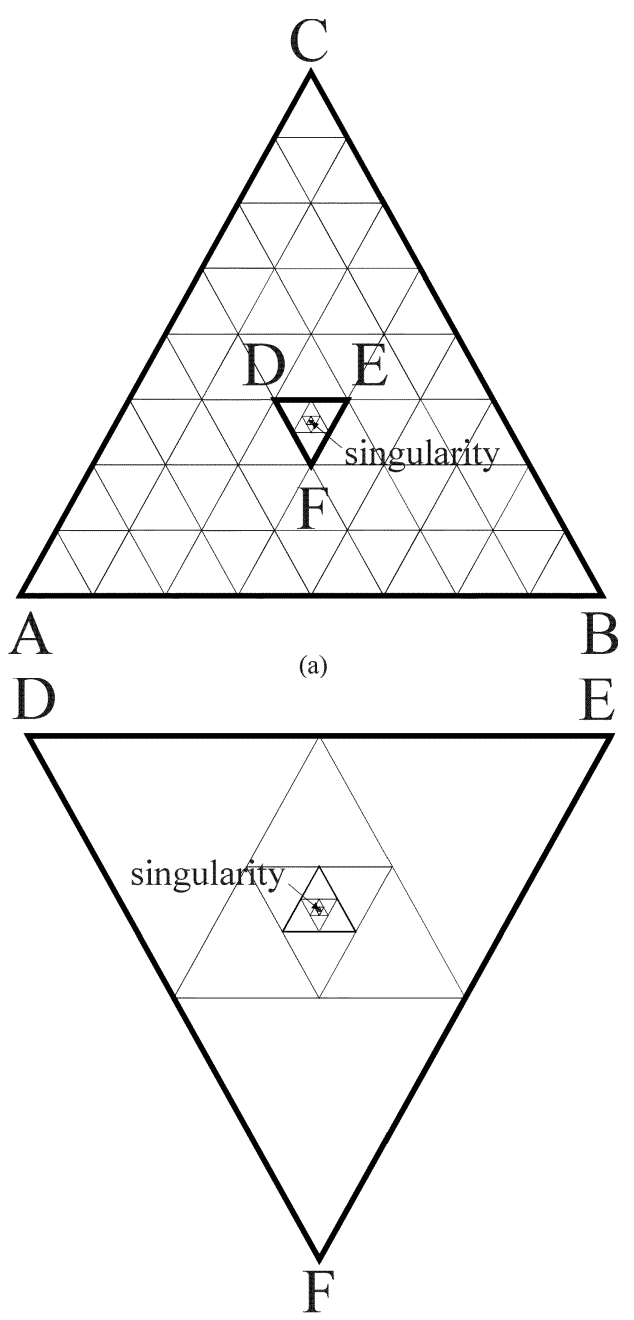

(b)

Fig. 2. Recursive subdivisions of a triangular domain to evaluate an integral with singularity located at the centroid. In this example, (a) the outer triangle $(\mathrm{ABC})$ is recursively subdivided three times while (b) the inner triangle (DEF) is subdivided six times in its central triangles only.

and more rapidly at points closer to the centroid, in this region recursive subdivisions are continued only in the central triangles (where the singularity is located), as indicated in Fig. 2(b). Typically, ten to 30 subdivisions of the central triangles would be required to accurately capture the variation of the integrand near the singularity. In this way, accurate evaluation can be performed much faster since only the central triangles of the inner triangle are continuously subdivided until the requested accuracy is reached. Alternatively, when a higher degree of accuracy is desired one can define an intermediate triangle between triangles $\mathrm{ABC}$ and DEF and performs more subdivisions only in that triangle.

As an illustration, in Table I the method is applied in the computation of the input impedance of a horizontal wire dipole above a lossy ground. Both the traditional and the proposed methods use quadratic quadrature rules and recursive subdivisions without singularity extractions. The result of the Numerical Electromagnetics Code (NEC-2) is shown as a comparison since it solves such a problem accurately. It can be seen that the results by the traditional method approach that of the proposed
TABLE I

NUMERICAL INTEGRATION METHODS

\begin{tabular}{cccc}
\hline \hline Method & No. of subdivisions & $\begin{array}{c}\text { Input } \\
\text { impedance } \\
\text { (Ohms) }\end{array}$ & $\begin{array}{c}\text { Time } \\
\text { (minutes) }\end{array}$ \\
\hline Traditional & 3 & $375.8-320.9 \mathrm{j}$ & $0: 3.67$ \\
Traditional & 5 & $385.3-330.5 \mathrm{j}$ & $0: 12.34$ \\
Traditional & 8 & $387.1-332.3 \mathrm{j}$ & $5: 21.20$ \\
This work & 3 (outer triangle) & $415.7-343.0 \mathrm{j}$ & $0: 4.11$ \\
& 30 (inner triangle) & & \\
NEC & - & $420.5-356.2 \mathrm{j}$ & - \\
\hline \hline
\end{tabular}

Numerical integration methods implemented in the computation of the input impedance of a horizontal wire dipole above a ground with $\varepsilon_{\mathrm{r}}=4$ and $\sigma=0.004 \mathrm{~S} / \mathrm{m}$. The dipole length $=50 \mathrm{~cm}$, wire diameter $=2 \mathrm{~mm}$ and elevation $=5 \mathrm{~mm}$. Computed for frequency $=1 \mathrm{GHz}$ and number of unknowns $=97$ on a $1.7-\mathrm{GHz}$ PC. The dipole is modeled as a thin strip in the first 4 rows and thin wire in the last one.

method (which is very close to NEC's result) as the number of subdivisions increases at the expense of longer computation time. On the other hand, the proposed method requires only slightly longer computation time (4.11 s) than the traditional one with three subdivisions $(3.67 \mathrm{~s})$. This shows that the addition of the inner triangle does not significantly increase computation time, yet it substantially improves accuracy. In [15] the traditional method is enhanced with a singularity extraction scheme which basically has a similar function as the inclusion of the inner triangle in the proposed method. As the evaluation time of the inner triangles is very short ( $0.44 \mathrm{~s}$ in the above example), we are confident that the overall computation times required by the proposed method and the one in [15] are at least comparable. We note that the main advantage of the proposed method is that no additional formulations (i.e., singularity extraction schemes) are required.

4) Interpolation Method for Sommerfeld Integrals: The fourth factor is to expedite the computation of Sommerfeld integrals using interpolation of pre-computed Sommerfeld integral values. In this work Sommerfeld integrals are pre-computed at a number of discrete distances $\left|\mathbf{r}-\mathbf{r}^{\prime}\right|$ for a given antenna elevation and saved in a table. Hence, any Sommerfeld integral values at an arbitrary distance within the range of the table can immediately be obtained using a table-lookup algorithm and interpolation.

5) Implementation of a Fast Frequency-Sweep Method: In addition to the four factors mentioned above, it is worth noting that the implementation of a fast frequency-sweep method such as the impedance-matrix interpolation technique in [17], can be an additional factor for further increasing the computation efficiency. This approach has been successfully implemented in the computation of the input impedance of complex antennas over an ultrawide frequency range, using MoM with triangular discretization [18]. Further development of the code employed in this work will include the implementation of an improved fast frequency-sweep method introduced in [19].

\section{Time-Domain Analysis}

The generator we use in the experiments produces a monocycle, which resembles the first derivative of a Gaussian 
pulse, with duration of $0.8 \mathrm{~ns}$. To simulate the wire bow-tie antenna excited by this monocycle we carry out computations in the frequency domain from $50 \mathrm{MHz}-5 \mathrm{GHz}$ with $50-\mathrm{MHz}$ frequency step, resulting in 100 frequency-domain data points. The $50 \mathrm{MHz}-5 \mathrm{GHz}$ range is chosen here because the spectral content of the 0.8-ns monocycle is meaningful only within this range. The inverse Fourier transformation is eventually employed to obtain the time-domain solution.

To obtain the characteristic impedance of an antenna, which is important for transient applications, one needs to compute the spectral feed current by assuming the antenna as being infinite. To calculate infinite-antenna feed current $I_{\infty}$ we employ the Fourier transform and its inverse as well as time windowing to exclude antenna internal reflections, which can be concisely written as

$$
I_{\infty}(\omega)=F\left\{W\left\{F^{-1}[I(\omega) S(\omega)]\right\}\right\}
$$

where $I$ is the spectrum of the feed current computed by the employed MoM scheme with 1 volt input voltage, and $S$ is the normalized spectrum of the input voltage pulse $V$. In (17) F, $\mathrm{F}^{-1}$ and $\mathrm{W}$ are the Fourier transformation, the inverse Fourier transformation and the time-window operators, respectively.

To exclude internal reflections from the time-domain response, in this work we introduce a time window with simple truncation process given by

$$
\begin{aligned}
& A_{k}=w A_{k+1}, k=-1,-2, \ldots \text { for the left end } \\
& A_{k}=w A_{k-1}, k=1,2, \ldots \text { for the right end }
\end{aligned}
$$

where $A$ is the value of the real or imaginary part of the signal, $A_{0}$ is the value of the signal at either the left or right end of the time window, and $w$ is a factor which determines the decay rate of the truncation process and assumes values in the range of $0 \leq w<1$. Typically, the most satisfactory results could be obtained with $w=0.7$ by applying the above equations to both of the real and imaginary parts of the signal separately. Note that when $w=0$ (18) reduces to a rectangular time window, which would result in the worst performance. Finally, using (17) and (18) the antenna characteristic impedance can be written as

$$
Z_{\infty}(\omega)=\frac{V(\omega)}{I_{\infty}(\omega)}
$$

\section{NUMERICAL AND EXPERIMENTAL RESUltS}

\section{A. Measurement Setup}

The measurements are carried out at our GPR test facility which consists of a pulse generator, a sampling scope, a large wooden sandbox and a scanner [20], [21]. The pulse generator produces the 0.8 -ns monocycle and the scanner accurately positions the antenna above the sand surface. The experimental antenna is fixed by a PVC support, which consists of a part that supports the wires and a pipe that supports the whole antenna as shown in Fig. 1(b). Each arm of the antenna comprises eight wire elements with $25-\mathrm{cm}$ length and $2-\mathrm{mm}$ diameter. The elements are $10^{\circ}$ separated from their adjacent neighbors to form a bow-tie flare angle of $70^{\circ}$. To measure the fields radiated into the ground, we use an electromagnetic probe [22] buried $16 \mathrm{~cm}$ below the surface.
To properly measure balanced loads, a balun is required. However, ultrawide-band baluns are not easy to produce and commercially available ones are expensive. Here, a technique to measure the input impedance of balanced antennas without baluns similar to that introduced in [23] is used. The antenna is fed using two identical 50-Ohm semi-rigid cables which are soldered together over their length, except for a small part near the ends where SMA connectors are attached. Each of the inner conductors of the other ends is soldered to one of the antenna terminals. The semi-rigid cables are connected by $50-\mathrm{Ohm}$ coaxial cables to the ports of a standard network analyzer, which has been previously calibrated at the SMA connectors. Post processing of the measured data is performed to remove the influence of the semi-rigid cables and to extract the desired differential-mode currents.

\section{B. Input Impedance}

The measurements of input impedance are carried out in the frequency domain. The measured frequency-domain data are Fourier transformed to the time-domain and subsequently time window (18) is employed to remove reflections from surrounding objects. Transformation back to the frequency domain is then performed to obtain the actual impedance of the antenna.

The measured and computed input impedance of the wire bow-tie antenna situated horizontally above dry sand is shown in Fig. 3 for $5-\mathrm{cm}$ and $1-\mathrm{cm}$ elevations. A previous measurement of the dielectric permittivity of the sand has led to $\varepsilon_{\mathrm{r}}=2.5$ for the sand relative permittivity, while for the sand conductivity we assume $\sigma=0.004 \mathrm{~S} / \mathrm{m}$. The computed results by NEC (which uses the thin-wire approximation) are included as a comparison. It can be seen that our computation (RWG-MoM) generally agrees with NEC. However, the difference in the model of the feed region most likely causes the discrepancy of the reactance for high frequencies seen in the figure. The feed region is modeled for the RWG MoM as a small bow-tie plate where the wires meet, while it is modeled for NEC as point-like wire junctions. Thus, the former model tends to be more capacitive than the latter especially for high frequencies. We note that as the feed-region model of the RWG-MoM approximates the actual feed region of the experimental antenna [see Fig. 1(a)] better than that of NEC, the RWG-MoM leads to better agreement with measurement for the reactance, as shown in Fig. 3.

Using (19) the characteristic impedance of the horizontal wire bow-tie antenna is computed as a function of elevation. The results are presented in Fig. 4, which generally also shows agreement between the RWG-MoM and NEC. However, for low elevations there is a slight discrepancy for the reactance which could be attributed to the difference of the feed-region models. Furthermore, it is worth noting that the ground influence on the input impedance is in this case significant only for very small distances (i.e., less than one fifth of the wavelength corresponding to the central frequency of the exciting pulse) from the interface.

Unfortunately, in Figs. 3 and 4 a discrepancy is observed between computation and measurement, which is caused most likely by the PVC support in the feed region that cannot be accounted for by both numerical methods. This discrepancy can be 


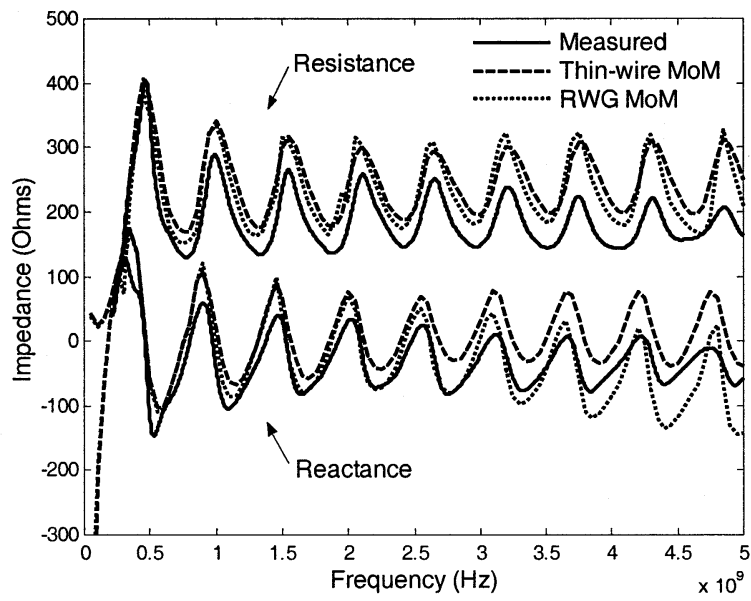

(a)

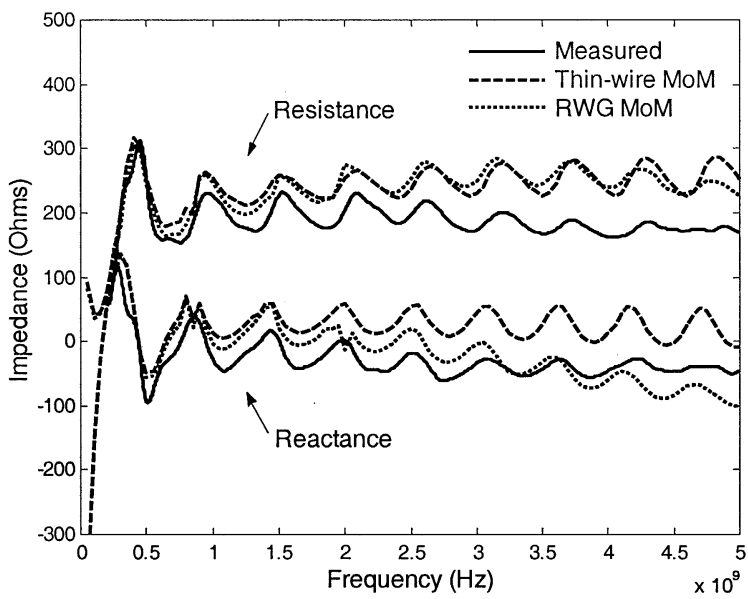

(b)

Fig. 3. Input impedance of the wire bow-tie antenna in Fig. 1. The ground, which is the dry sand, is modeled with $\varepsilon_{\mathrm{r}}=2.5$ and $\sigma=0.004 \mathrm{~S} / \mathrm{m}$. The antenna elevation is (a) $5 \mathrm{~cm}$ and (b) $1 \mathrm{~cm}$.

more clearly seen in Fig. 4. The influence of the PVC support for the antenna in free space has been numerically analyzed using the commercial MoM package FEKO, and it has been found that the PVC support reduced the resistance by about $50 \Omega$ [24], similar to our result in Fig. 4.

Moreover, we perform a numerical experiment to study the influence of flare angle variation on the wire bow-tie's input impedance. For this purpose we model four different wire bow-tie antennas with flare angles of $25^{\circ}, 75^{\circ}, 125^{\circ}$, and $175^{\circ}$, formed by respectively two, four, six, and eight elements in each arm. The characteristic impedance of the antennas is plotted in Fig. 5 for different ground types. It can be seen that unlike solid bow ties which exhibit zero characteristic reactance [2], wire bow-tie antennas have nonzero characteristic reactance which is an inherent property of wire structures [25]. Futhermore, Fig. 5 indicates that the largest possible flare angle provides the best matched condition for a $50-\Omega$ feed line.

\section{Transmit Waveform}

The waveform transmitted into the ground is another important antenna property, which describes the transient behavior of the antenna. For applications like short-range radars (including

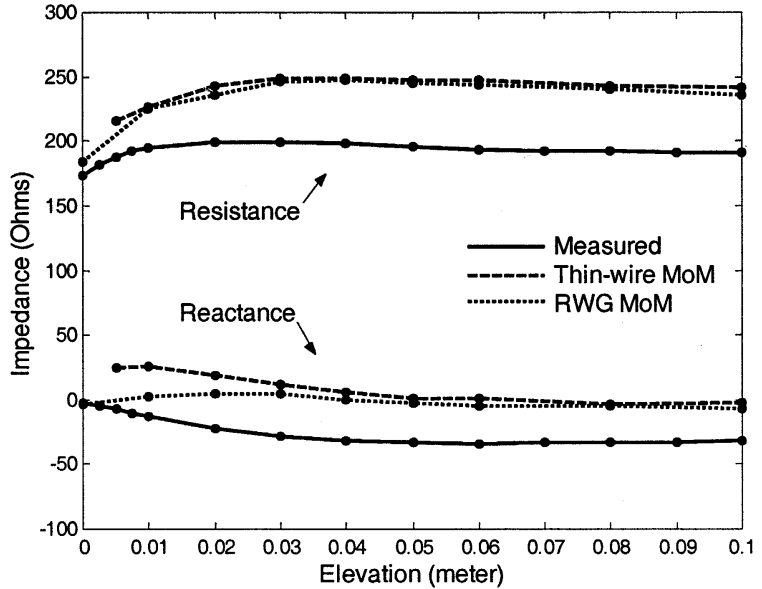

Fig. 4. Characteristic impedance of the wire bow-tie antenna in Fig. 1 with respect to the 0.8 -ns monocycle, as a function of elevation above the dry sand.

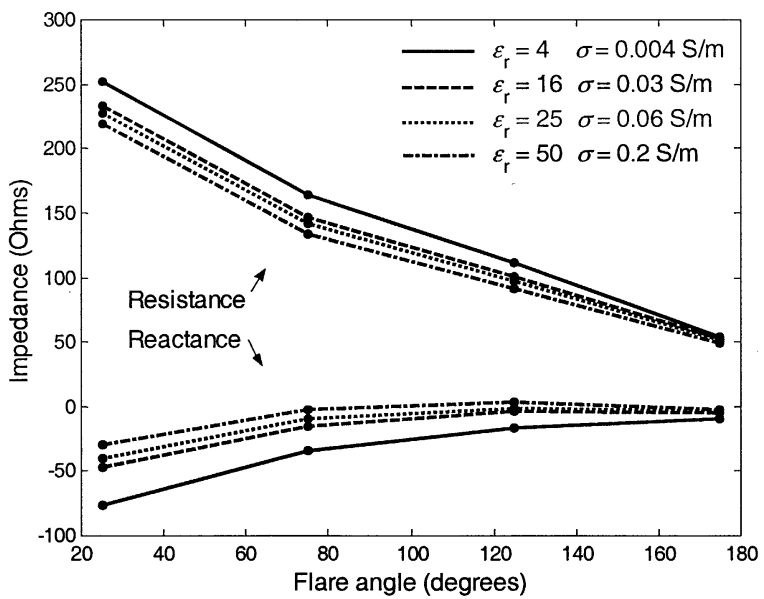

Fig. 5. Characteristic impedance of horizontal wire bow-tie antennas with respect to the 0.8-ns monocycle, as a function of flare angle, computed for a flare angle of $25^{\circ}, 75^{\circ}, 125^{\circ}$, and $175^{\circ}$ with respectively two, four, six, and eight elements in each arm. The antenna is elevated $5 \mathrm{~mm}$ above a half-space with different electrical properties.

GPRs) it is important to have minimal late-time ringing in the transmit waveform. Such ringing increases clutter level in the measured target response.

The normalized subsurface transmit waveform at a $16-\mathrm{cm}$ depth (in the dry sand) in the broadside direction of the horizontal wire bow-tie antenna is computed and measured in Fig. 6. The difference in the shape of the computed and measured waveforms is due to the fact that in this case what we measure is the induced voltage at the terminal of the probe (in volts), while what we compute is the electric field at the probe's location (in volts/meter). The measured curve is naturally affected by the characteristics of the probe in the ground. To remove the probe's influence on the measurement, a code for deconvolving the probe characteristics and measured data has been developed based on [22]. The code is here employed to obtain the deconvolved antenna response presented in Fig. 6, which reveals the influence of the PVC support (see Fig. 1(a) in the form of a reflection from the support's ends. This reflection appears $0.3 \mathrm{~ns}$ after the main pulse and in Fig. 6 the maximum 


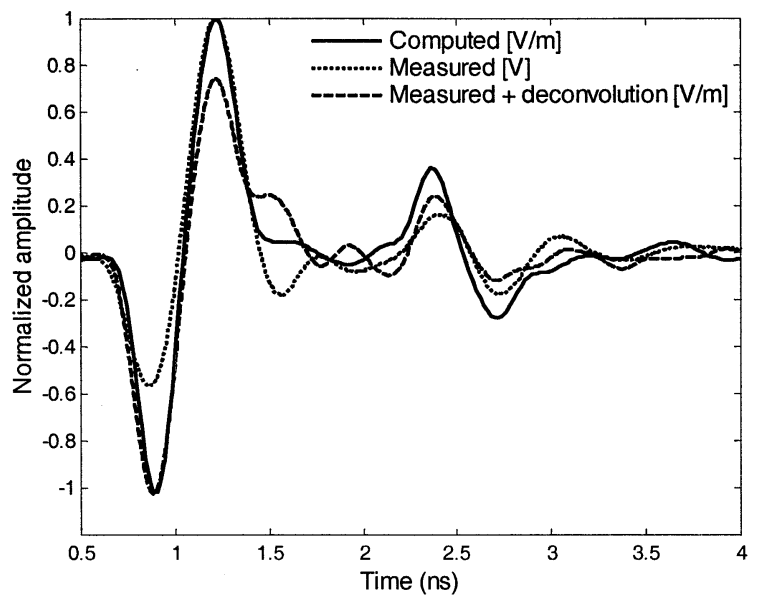

Fig. 6. Normalized subsurface transmit waveform (in the dry sand, $16 \mathrm{~cm}$ below the interface, in the broadside direction) of the wire bow-tie antenna in Fig. 1, measured in volt (induced voltage at the probe's terminal) and computed in volt/meter (electric field at the probe's location). Deconvolution of the probe characteristics and measured data is employed to remove the probe's influence. Antenna elevation $=5 \mathrm{~mm}$.

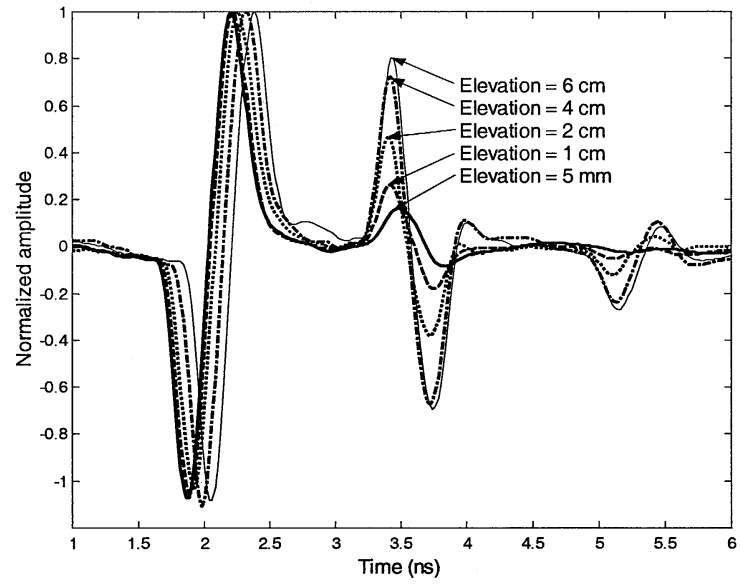

Fig. 7. Normalized subsurface transmit waveforms $(16 \mathrm{~cm}$ below the interface, in the broadside direction) of the wire bow-tie antenna in Fig. 1, computed for different elevations above the interface. The assumed ground parameters are $\varepsilon_{\mathrm{r}}=4$ and $\sigma=0.004 \mathrm{~S} / \mathrm{m}$.

of the reflection is visible at about $1.5 \mathrm{~ns}$. The minimum of the reflection comes before the maximum and reduces the maximum of the main pulse at about $1.2 \mathrm{~ns}$. It should be noted that the deconvolution scheme assumes a normal plane-wave incidence and hence it might introduce inaccuracy for the pulse radiated from the antenna ends, as it arrives at the probe at an oblique incidence.

The subsurface transmit waveforms at a $16-\mathrm{cm}$ depth in the broadside direction of the wire bow-tie antenna are computed for different antenna elevations, and normalized in Fig. 7. The ground is modeled as a half-space with $\varepsilon_{\mathrm{r}}=4$ and $\sigma=$ $0.004 \mathrm{~S} / \mathrm{m}$. It has been found that the amplitude of the waveforms in Fig. 7 increases for lower elevations due to stronger antenna-ground coupling as the antenna moves closer to the ground. In the figure it can be seen that because of its finite conductivity the ground obviously acts as a resistive loading since it reduces end reflections as the antenna approaches the interface.

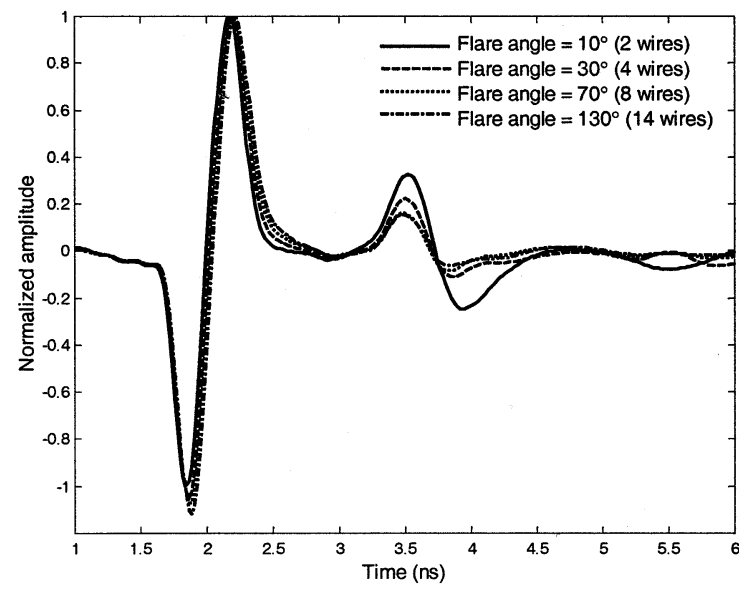

Fig. 8. Normalized subsurface transmit waveforms $(16 \mathrm{~cm}$ below the interface, in the broadside direction) of horizontal wire bow-tie antennas (length $=50 \mathrm{~cm}$, wire diameter $=2 \mathrm{~mm}$ ), computed for different flare angles. Antenna elevation $=5 \mathrm{~mm}$ and the assumed ground parameters are $\varepsilon_{\mathrm{r}}=4$ and $\sigma=0.004 \mathrm{~S} / \mathrm{m}$.

We can also see that the ground influence is significant only for small elevations of the order of a few centimeters from the interface. This result demonstrates the absorbing property of a lossy ground and suggests that late-time ringing would be less a problem when the antenna is situated very close to the interface.

The waveforms at the same depth in the ground are computed for wire bow-tie antennas with different flare angles situated $5 \mathrm{~mm}$ above the interface, and normalized in Fig. 8. The considered flare angles are $10^{\circ}, 30^{\circ}, 70^{\circ}$, and $130^{\circ}$, formed by respectively two, four, eight, and 14 wires in each of the bow-tie arms with $10^{\circ}$ separation between two neighboring wires. We have observed that the amplitude of the waveforms increases as the flare angle is enlarged. By normalizing the waveforms in Fig. 8 it is evident that increasing the flare angle improves the antenna bandwidth. This is shown by the decrease in the level of the reflections as the flare angle becomes larger. However, it can be seen that the level of the reflections starts to stabilize when the flare angle is $70^{\circ}$.

\section{Antenna Footprint}

An antenna footprint is defined as a distribution of the peak values of transmit waveforms within a horizontal plane on the ground surface or subsurface. It indicates the shape and size of the spot illuminated by the antenna. In many GPR applications, antenna footprints play an important role since radar imaging can be improved when the shape and size of the footprint are comparable to those of the targets. When the footprint is too large it gives rise to ground clutters. On the other hand, when the footprint is too small the resulting hyperbola in a radar B-scan might be too small for detection. Moreover, an optimal footprint is important for improving target localization. For long targets like buried pipes or cables, it is desired to have a footprint with elongated shape, while for circular targets such as landmines, a footprint with circular shape would be preferred. In this work the footprint measurement is carried out on a horizontal plane at a 16-cm depth and the result is plotted in Fig. 9(a). The computed footprint is shown in Fig. 9(b), which predicts the measured result. 


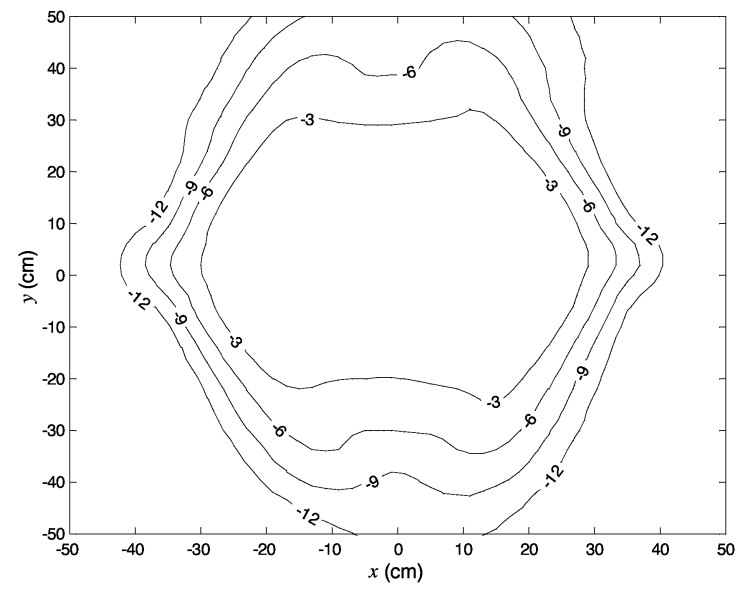

(a)

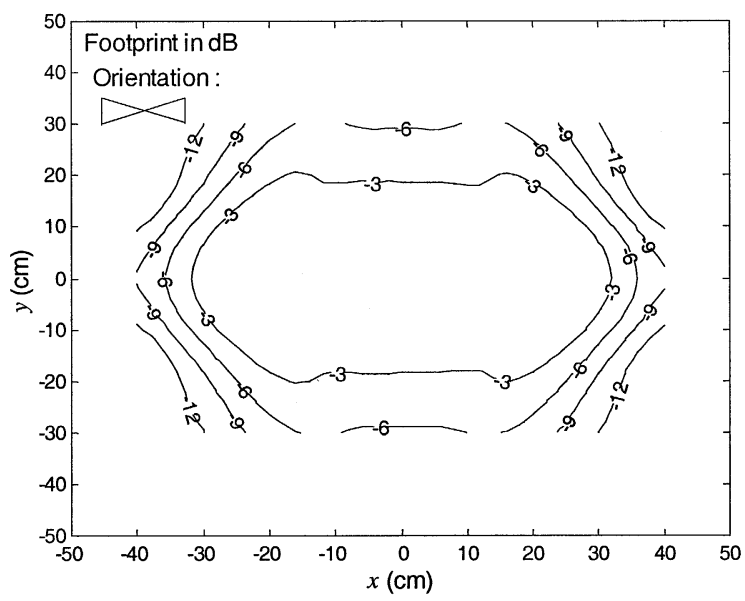

(b)

Fig. 9. (a) Measured and (b) computed subsurface footprint (in the dry sand, $16 \mathrm{~cm}$ below the interface, in decibels) of the horizontal wire bow-tie antenna in Fig. 1. Antenna elevation $=2 \mathrm{~cm}$.

In Fig. 10 we present the magnitude of the spectrum of the transmit waveform at observation points along the main axes of the wire bow-tie antenna for observations at a 16-cm depth from the interface. In addition to the antenna footprints discussed previously, the results in Fig. 10 offer the opportunity to perform an in-depth analysis of the antenna frequency characteristics as a function of space. For example, in both of Fig. 10(a) and (b) the resonance behavior of the antenna are evident at the center of the footprint (at $0 \mathrm{~cm}$ ) where the peaks and the deeps in the spectrum can clearly be seen. As the observation is shifted along the axes, the spectrum of the transmit waveform changes due to interference between the radiation from the feed point and antenna ends. It can be seen that the locations of the peaks form a hyperbola for observations near the center of the footprint. However, in Fig. 10(a) we can see that as the observation moves toward the antenna ends (at $25 \mathrm{~cm}$ from the center), an abrupt change of the peaks' location occurs at about $13 \mathrm{~cm}$ from the center of the footprint, where the highest peaks are abruptly shifted from about $1.4 \mathrm{GHz}$ to about $800 \mathrm{MHz}$. This result indicates a significant difference between the radiation characteristics of the feed point and antenna ends, as the frequency content of the

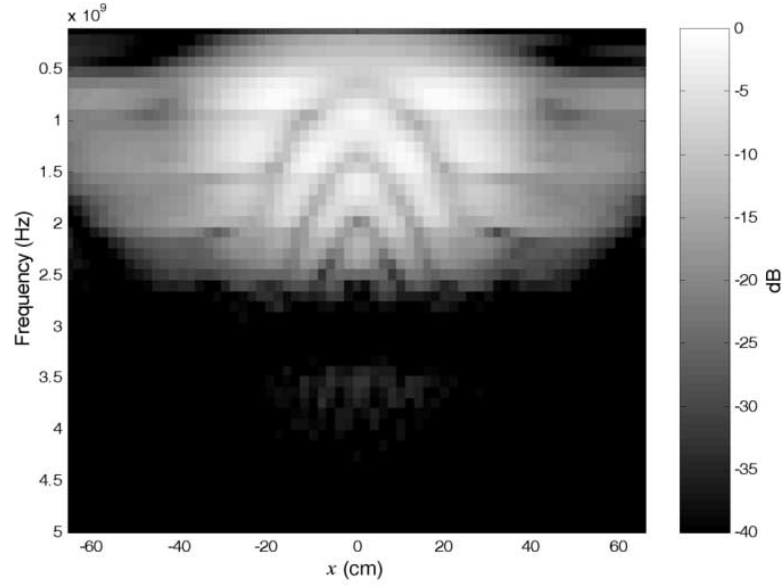

(a)

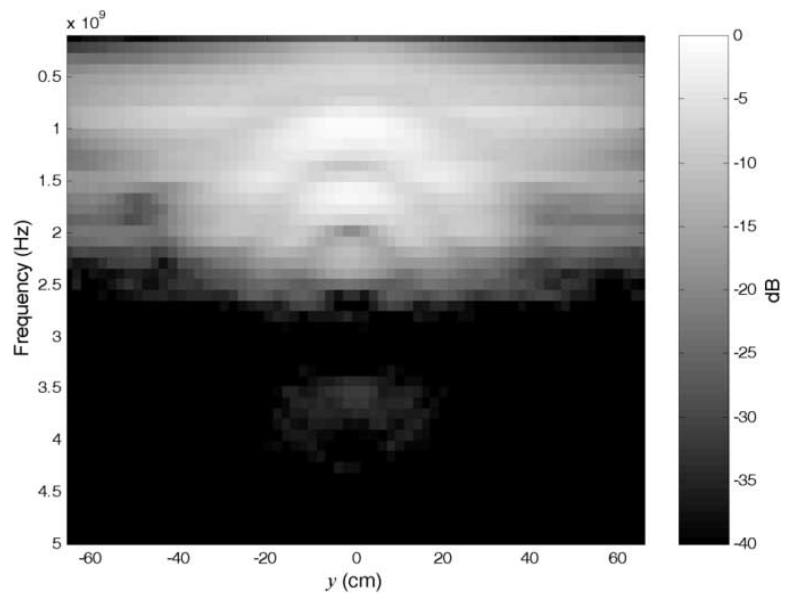

(b)

Fig. 10. Measured spectrum of the transmit waveform (in the dry sand, $16 \mathrm{~cm}$ below the interface, in decibels) of the wire bow-tie antenna in Fig. 1 as a function of displacement along (a) the $x$ axis and (b) the $y$ axis. Antenna elevation $=2 \mathrm{~cm}$.

exciting pulse below $1 \mathrm{GHz}$ is radiated more efficiently by the ends, while higher frequencies are radiated more efficiently by the feed region.

Additionally, we perform a numerical experiment of two extreme cases when the flare angle of the wire bow-tie antenna is very small and very large. Fig. 11(a) presents the computed footprint of a horizontal wire bow-tie antenna with $10^{\circ}$ flare angle consisting of 2 elements in each arm. It is shown that in this case the footprint has an elongated shape, which would be suitable for detecting and locating long objects. Fig. 10(b) presents the footprint of a wire bow-tie antenna with $150^{\circ}$ flare angle consisting of 16 elements in each arm. It is shown that in this case the shape of the footprint becomes more circular, preferred for detecting and localizing round or spherical objects.

\section{CONCLUSION}

In this paper we analyze the performance of a circular-end wire bow-tie antenna theoretically and experimentally. Using the mixed-potential integral equation formulation and modeling de wires as a tape-like structure, we develop a numerical model 


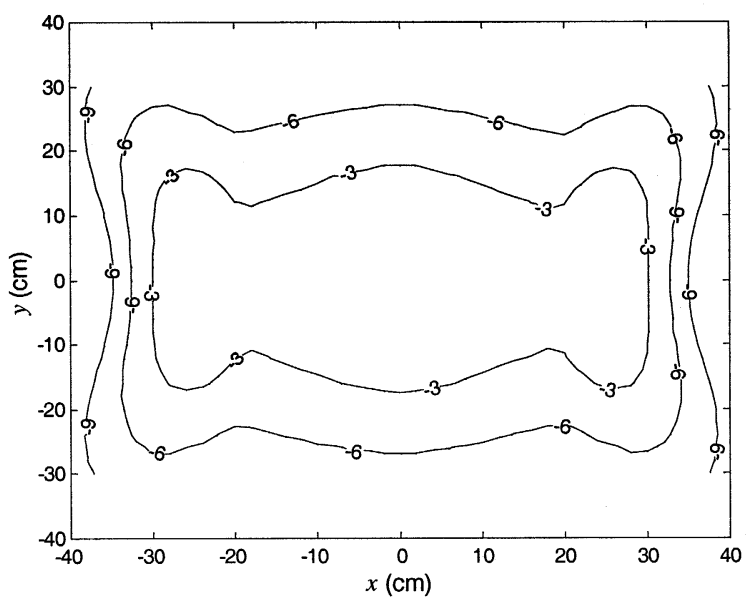

(a)

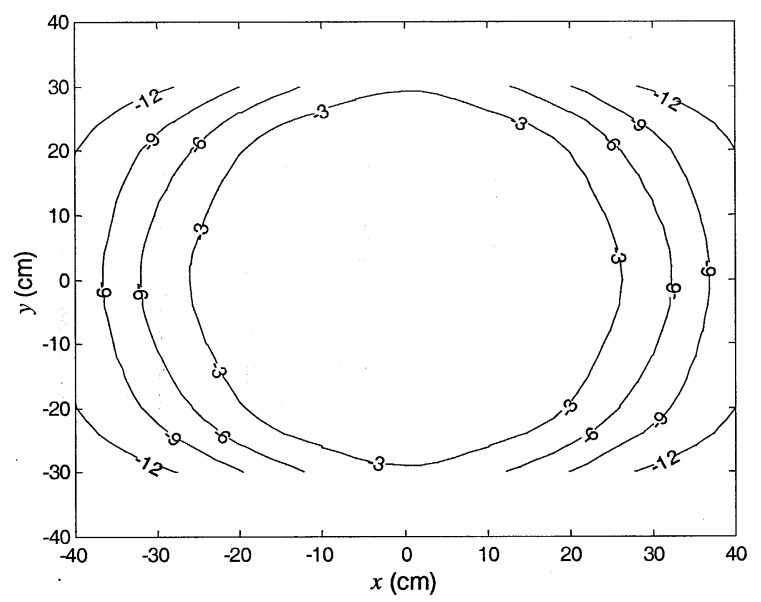

(b)

Fig. 11. Computed subsurface footprints $(16 \mathrm{~cm}$ below the interface, in decibels) of a horizontal wire bow-tie antenna with two elements in each arm forming a flare angle of $10^{\circ}$, and (b) the antenna has 16 elements in each arm forming a flare angle of $150^{\circ}$. Antenna elevation $=2 \mathrm{~cm}$. The ground is the dry sand modeled with $\varepsilon_{\mathrm{r}}=2.5$ and $\sigma=0.004 \mathrm{~S} / \mathrm{m}$.

of the antenna. The advantage of this model over the NEC-2 model is that its accuracy does not deteriorate when the antenna approaches the ground. We pay a special attention to efficient numerical realization of the model as we plan to use the developed code for simulation of much more complicated antennas. We verify the simulation results of the model with experimental data as well as NEC-2 results. We analyze the characteristic impedance, the transmit waveform, and the footprint of the antenna for different flare angles, elevations above the ground and ground types. We observe that the ground affects the antenna performance only for very small distances (i.e., less than one fifth of the wavelength corresponding to the central frequency of the exciting pulse) from the interface. Moreover, the presence of the ground decreases the antenna resistance and late-time ringing. We point out that matching the antenna to the feed line for different ground types and antenna elevations can be done by varying the flare angle. Furthermore, we also demonstrate a possibility of footprint adjustment by flare angle variation.

\section{ACKNOWLEDGMENT}

The authors thank Prof. K. D. Palmer from Stellenbosch University, South Africa, for his invaluable suggestions, P. Hakkaart for his assistance in the construction of the experimental antenna and J. Zijderveld for his assistance in the measurements. The authors also thank the anonymous reviewers for their constructive comments.

\section{REFERENCES}

[1] G. H. Brown and O. M. Woodward Jr., "Experimentally determined radiation characteristics of conical and triangular antennas," RCA Rev., vol. 13, pp. 425-452, Dec. 1952

[2] R. L. Carrel, "The characteristic impedance of two infinite cones of arbitrary cross section," IRE Trans. Antennas Propagat., vol. AP-6, pp. 197-201, Apr. 1958.

[3] K. L. Shlager, G. S. Smith, and J. G. Maloney, "Optimization of bow-tie antennas for pulse radiation," IEEE Trans. Antennas Propagat., vol. 42, pp. 975-982, July 1994.

[4] C. J. Leat, N. V. Shuley, and G. F. Stickley, "Triangular-patch model of bowtie antennas: Validation against Brown and Woodward," Proc. Inst. Elect. Eng. Microwave Antennas Propagation, vol. 145, no. 6, pp. 465-470, Dec. 1998.

[5] Y. Nishioka, O. Maeshima, T. Uno, and S. Adachi, "FDTD analysis of resistor-loaded bow-tie antennas covered with ferrite-coated conducting cavity for subsurface radar," IEEE Trans. Antennas Propagat., vol. 47, pp. 970-977, June 1999.

[6] C. J. Leat, "A moment method model for ground penetrating radar antennas," in Proc. Millenium Conf. Antennas Propagation (AP-2000), Davos, Switzerland, Apr. 2000, CD-ROM.

[7] C. E. Smith, C. M. Butler, and K. R. Umashankar, "Characteristics of a wire biconical antenna," Microw. J., vol. 22, no. 9, pp. 37-40, Sept. 1979.

[8] A. A. Lestari, A. G. Yarovoy, and L. P. Ligthart, "Characterization of transient bow-tie antennas for ground penetrating radar," in Proc. 9th Int. Conf. Ground Penetrating Radar, Santa Barbara, CA, Apr. 2002, pp. $80-85$.

[9] K. A. Michalski and D. Zheng, "Electromagnetic scattering and radiation by surfaces of arbitrary shape in layered media, Part I: Theory; Part II: Implementation and results for contiguous half-spaces," IEEE Trans. Antennas Propagat., vol. 38, pp. 335-352, Mar. 1990.

[10] S. M. Rao, D. R. Wilton, and A. W. Glisson, "Electromagnetic scattering by surfaces of arbitrary shape," IEEE Trans. Antennas Propagat., vol. AP-30, pp. 409-418, May 1982.

[11] C. M. Butler, "The equivalent radius of a narrow conducting strip," IEEE Trans. Antennas Propagat., vol. AP-30, pp. 755-758, July 1982.

[12] W. A. Johnson, D. R. Wilton, and R. M. Sharpe, "Modeling scattering from and radiation by arbitrary shaped objects with the electric field integral equation triangular surface patch code," Electromagn., vol. 10, pp. 41-63, 1990.

[13] O. C. Zienkiewicz, The Finite Element Method in Engineering Science. London, U.K.: McGraw-Hill, 1971.

[14] A. Moumen, "Analysis and synthesis of compact feeds for large multiple-beam reflector antennas," Ph.D. dissertation, Delft Univ. Technol., The Netherlands, 2000.

[15] D. R. Wilton et al., "Potential integrals for uniform and linear source distributions on polygonal and polyhedral domains," IEEE Trans. Antennas Propagat., vol. AP-32, pp. 276-281, Mar. 1984.

[16] S. Caorsi, D. Moreno, and F. Sidoti, "Theoretical and numerical treatment of surface integrals involving the free-space green's function," IEEE Trans. Antennas Propagat., vol. 41, pp. 1296-1301, Sept. 1993.

[17] E. H. Newman, "Generation of wide-band data from the method of moments by interpolating the impedance matrix," IEEE Trans. Antennas Propagat., vol. AP-36, pp. 1820-1824, Dec. 1988.

[18] K. L. Virga and Y. Rahmat-Samii, "Efficient wide-band evaluation of mobile communications antennas using $[\mathrm{Z}]$ or $[\mathrm{Y}]$ matrix interpolation with the method of moments," IEEE Trans. Antennas Propagat., vol. 47, pp. 65-76, Jan. 1999.

[19] J. E. Bracken, D.-K. Sun, and Z. J. Cendes, "S-domain methods for simultaneous time and frequency characterization of electromagnetic devices," IEEE Trans. Microwave Theory Tech., vol. 46, pp. 1277-1290, Sept. 1998. 
[20] A. Schukin, I. Kaploun, A. Yarovoy, and L. Ligthart, "Evolution of GPR antennas, pulse generators, and sample recorders," presented at the Millennium Conf. Antennas \& Propagation (on CD-ROM), Davos, Switzerland, Apr. 9-14, 2000.

[21] A. G. Yarovoy, P. J. Aubry, and L. P. Ligthart, "GPR antenna measurements in time domain," presented at the Proc. Millenium Conf. Antennas Propagat. (on CD-ROM), Davos, Switzerland, Apr. 9-14, 2000.

[22] A. Yarovoy, R. de Jongh, and L. Ligthart, "Ultra-wideband sensor for electromagnetic field measurements in time domain," Electron. Lett., vol. 36, no. 20, pp. 1679-1680, Sept. 2000.

[23] K. D. Palmer and M. W. van Rooyen, "Simple broadband measuremen of balanced loads using a network analyzer," presented at the Proc. Millenium Conf. Antennas Propagat. (on CD-ROM), Davos, Switzerland, Apr. 9-14, 2000.

[24] K. D. Palmer, private communication, 2002.

[25] T. T. Wu, "Input admittance of infinitely long dipole antennas driven from coaxial lines,” J. Math. Phys., vol. 3, pp. 1298-1301, 1962.

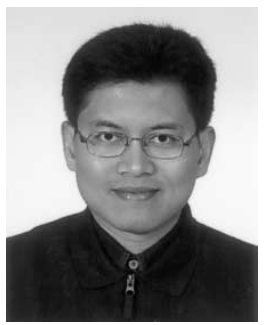

Andrian Andaya Lestari was born in Bogor, Indonesia. He received the ingenieur and Ph.D. degrees in electrical engineering from Delft University of Technology, Delft, The Netherlands, in 1993 and 2003, respectively.

From 1993 to 1998 , he was with a government research agency in Jakarta, Indonesia. He joined the International Research Centre for Telecommunications-transmission and Radar (IRCTR), Delft University of Technology, as a researcher in 1998. His work at IRCTR has resulted in over 20 publications, which include national and international patents, journal and conference papers, and scientific reports. Currently he is active in the development of ultrawide-band antennas and numerical tools for transient antenna analysis.

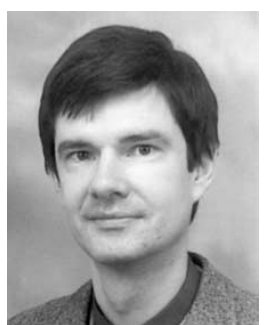

Alexander G. Yarovoy (M'96) received the Diploma (with honors) in radiophysics and electronics and the Cand. Phys. \& Math. Sci. and Dr. Phys. \& Math. Sci. degrees in radiophysics, from Kharkov State University, Kharkov, Ukraine, in 1984, 1987, and 1994, respectively.

In 1987, he joined the Department of Radiophysics, Kharkov State University, as a Researcher and became a Professor in 1997. From September 1994 through 1996, he was with the Technical University of Ilmenau, Germany, as a Visiting Researcher. Since 1999, he has been with the International Research Centre for Telecommunications-Transmission and Radar (IRCTR), Delft University of Technology, Delft, The Netherlands, where he coordinates all GPR-related projects. His main research interests are in ultrawide-band electromagnetics, wave scattering from statistically rough surfaces and penetrable obstacles and computational methods in electromagnetics.

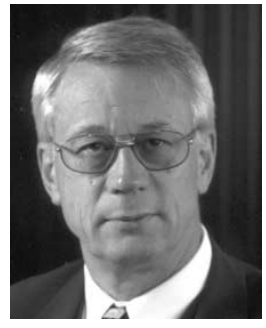

Leo P. Ligthart (M'94-SM'95-F'02) was born in Rotterdam, the Netherlands, on September 15, 1946. $\mathrm{He}$ received the Engineer's degree (cum laude) and the Doctor of Technology degree from Delft University of Technology, Delft, The Netherlands, in 1969 and 1985, respectively, the Doctorates (honoris causa) from Moscow State Technical University of Civil Aviation, Moscow, Russia, in 1999, and the Doctorates (honoris causa) from Tomsk State University of Control Systems and Radioelectronics, Tomsk, Russia, in 2001.

Since 1992, he has held the Chair of Microwave Transmission, Radar and Remote Sensing in the Department of Information Technology and Systems, Delft University of Technology, where in 1994, he became Director of the International Research Centre for Telecommunications-Transmission and Radar. His principal areas of specialization include antennas and propagation, radar and remote sensing, but he has also been active in satellite, mobile, and radio communications. 